JOURNAL OF THEORETICAL

AND APPLIED MECHANICS

55, 1, pp. 17-27, Warsaw 2017

DOI: $10.15632 /$ jtam-pl.55.1.17

\title{
MECHANICAL ANALYSIS OF AUTOFRETTAGED HIGH PRESSURE APPARATUS
}

\author{
RUILIN ZHU \\ College of Engineering and Design, Hunan Normal University, Changsha, China \\ e-mail:zrl200701@sina.com
}

\section{GuOLin Zhu}

Basic Courses Department, Jiangxi Police College, Nanchang, China

Aifeng MaO

Moshanjie School, Nanchang, China

High pressure apparatus is widely used in industries, the design of them depends on stress distributions in their walls. Most of high pressure apparatuses are made in form of cylinders. To raise load-bearing capacity and extend operation life for high pressure apparatus, the autofrettage technology is often used. To design autofrettaged high pressure apparatus, it is necessary to study characteristics of stresses in the wall of thick-wall cylinders, including residual stresses and total stresses, etc. In this study, through investigating the characteristics of stresses of cylinders subjected to internal pressure according to the maximum distortion strain energy theory, a set of simplified equations for residual stresses and total stresses are obtained, the safe and optimum load-bearing conditions for autofrettaged cylinders are found out, which are the basis for design of autofrettaged high pressure apparatus.

Keywords: thick-wall cylinder, autofrettage, total stress, residual stress, load-bearing capacity

\section{Nomenclature}

$r_{i}, r_{j}, r_{o} \quad-$ inside radius, radius of elastic-plastic juncture, outside radius, respectively

$k \quad-\quad$ ratio of outside to inside radius, $k=r_{o} / r_{i}$

$k_{j} \quad-$ depth of plastic zone or plastic depth, $k_{j}=r_{j} / r_{i}$

$k_{j^{*}} \quad-$ optimum $k_{j}$

$k_{c} \quad-\quad$ critical radius ratio, $k_{c}=2.2184574899167 \ldots$

$x \quad-\quad$ relative location, $x=r / r_{i}$

$p, p_{y} \quad-\quad$ internal and entire yield pressure

$p_{a} \quad-$ autofrettage pressure

$p_{e} \quad-\quad$ maximum elastic load-bearing capability of unautofrettaged cylinder or initial yield pressure;

$\sigma_{y} \quad-\quad$ yield strength

$\sigma_{e} \quad-$ equivalent stress

Superscripts

$p, t, 1$ - quantity related with internal pressure, total and residual stress, respectively. Subscripts

$z, r, \theta$ - axial, radial and circumferential direction, respectively. 


\section{Introduction}

Cylinders are widely used in manufacturing high and ultra-pressure vessels, high-pressure pumps, battleship and tank cannon barrels as well as fuel injection systems for diesel engines, etc. The autofrettage technique is an effective method to raise load-bearing capacity and extend operation life of cylinders. Usually, in the most commonly employed autofrettage process, a cylinder is pressurized to a quite high internal hydraulic pressure, as a result, the portion of the cylinder from inner radius to some intermediate radius becomes plastic while the remaining portion remains elastic. After releasing the pressure, the residual stresses are set up in the wall of the cylinder.

Studies on autofrettage about specific engineering problems have been done widely. Finite element simulations and experiments, the interaction between manufacturing processes with respect to residual stresses and deformations was studied by Brünnet and Bähre (2014). Farrahi et al. (2012) investigated the residual stress distribution at the wall of a thick-walled tube affected by the re-autofrettage process. The effects of thermal autofrettage on the residual stresses in a titanium-copper brazed joint were studied by Hamilton et al. (2015). Lin et al. (2009) built the autofrettage damage mechanics model from an ultra-high pressure vessel autofrettage damage mechanism. By using continuum damage mechanics approach, Lvov and Kostromitskaya (2014) analyzed the autofrettage process and derived general set of government equations of elasticplastic bodies by using the effective stress concept. A finite element model of the swaging process was developed in ANSYS and systematically refined to investigate the mechanism of deformation and subsequent development of residual stresses by Gibson et al. (2014). Noraziah et al. (2011a,b) set an analytical autofrettae procedure to predict the required autofrettage pressure for various levels of allowable pressure and to achieve maximum fatigue life. By using Huang's model for modeling reverse yielding due to Bauschinger effect, Bhatnagar (2013) presented an original concept of an autofrettage compounded tube which was modeled for the autofrettage process. By using the Kendall model, which was adopted by ASME Code, Shim et al. (2010) predicted the accurate residual stress of SNCM 8 high strength steel. Zheng and Xuan $(2010,2011)$ analyzed the optimum autofrettage pressure of a thick wall cylinder under thermo-mechanical loadings and investigated theoretically and validated by the finite element method (FEM) the closed form solution of the limit thermal load of autofrettage and the optimum autofrettage pressure under plane strain and open-ended conditions. Zhu (2008) investigated the optimum plastic depth and load-bearing capacity of an autofrettaged cylinder in terms of the point of view of avoiding compressive yield after removing autofrettage pressure and raising load-bearing capacity as far as possible simultaneously. Zhu and Zhu (2013) studied autofrettage of cylinders by limiting circumferential residual stress and according to Mises Yield criterion. Zhu and Li (2014) presented equations of optimum overstrain $\left(\varepsilon_{\lambda}\right)$ and depth of the plastic zone $\left(k_{j \lambda}\right)$ for a certain load-bearing capacity and radius ratio $(k)$.

This paper is intended to investigate the varying tendency and distribution laws of stresses in autofrettaged cylinders so as to provide the theoretic basis for the design of high pressure apparatus. Engineering conditions are in endless variety. This paper is based on ideal conditions including (1) the material of a cylinder is perfectly elastic-plastic and the Bauschinger effect is neglected, the compressive yield limit is equal to the tensile one; (2) strain hardening is ignored; (3) there is not any defect in the material.

\section{General residual stresses}

After removing autofrettage pressure, residual stresses remain in the wall of a cylinder. Yu (1980) put forward the residual stresses at a general radius location which has been re-arranged as follows: 
— in the plastic zone

$$
\begin{aligned}
& \frac{\sigma_{z}^{\prime}}{\sigma_{y}}=\frac{1}{\sqrt{3}}\left[\frac{k_{j}^{2}}{k^{2}}+\ln \frac{x^{2}}{k_{j}^{2}}-\left(1-\frac{k_{j}^{2}}{k^{2}}+\ln k_{j}^{2}\right) \frac{1}{k^{2}-1}\right] \\
& \frac{\sigma_{r}^{\prime}}{\sigma_{y}}=\frac{1}{\sqrt{3}}\left[\frac{k_{j}^{2}}{k^{2}}-1+\ln \frac{x^{2}}{k_{j}^{2}}-\left(1-\frac{k_{j}^{2}}{k^{2}}+\ln k_{j}^{2}\right) \frac{1}{k^{2}-1}\left(1-\frac{k^{2}}{x^{2}}\right)\right] \\
& \frac{\sigma_{\theta}^{\prime}}{\sigma_{y}}=\frac{1}{\sqrt{3}}\left[\frac{k_{j}^{2}}{k^{2}}+1+\ln \frac{x^{2}}{k_{j}^{2}}-\left(1-\frac{k_{j}^{2}}{k^{2}}+\ln k_{j}^{2}\right) \frac{1}{k^{2}-1}\left(1+\frac{k^{2}}{x^{2}}\right)\right]
\end{aligned}
$$

Accordiong to the Mises criterion, the equivalent residual stress is

$$
\frac{\sigma_{e}^{\prime}}{\sigma_{y}}=\frac{\sqrt{3}}{2}\left(\frac{\sigma_{\theta}^{\prime}}{\sigma_{y}}-\frac{\sigma_{r}^{\prime}}{\sigma_{y}}\right)=1-\frac{k^{2}-k_{j}^{2}+k^{2} \ln k_{j}^{2}}{\left(k^{2}-1\right) x^{2}}
$$

— in the elastic zone

$$
\begin{aligned}
& \frac{\sigma_{z}^{\prime}}{\sigma_{y}}=\frac{1}{\sqrt{3}}\left[\frac{k_{j}^{2}}{k^{2}}-\left(1-\frac{k_{j}^{2}}{k^{2}}+\ln k_{j}^{2}\right) \frac{1}{k^{2}-1}\right] \\
& \frac{\sigma_{r}^{\prime}}{\sigma_{y}}=\frac{1}{\sqrt{3}}\left(1-\frac{k^{2}}{x^{2}}\right)\left[\frac{k_{j}^{2}}{k^{2}}-\left(1-\frac{k_{j}^{2}}{k^{2}}+\ln k_{j}^{2}\right) \frac{1}{k^{2}-1}\right]=\left(1-\frac{k^{2}}{x^{2}}\right) \frac{\sigma_{z}^{\prime}}{\sigma_{y}} \\
& \frac{\sigma_{\theta}^{\prime}}{\sigma_{y}}=\frac{1}{\sqrt{3}}\left(1+\frac{k^{2}}{x^{2}}\right)\left[\frac{k_{j}^{2}}{k^{2}}-\left(1-\frac{k_{j}^{2}}{k^{2}}+\ln k_{j}^{2}\right) \frac{1}{k^{2}-1}\right]=\left(1+\frac{k^{2}}{x^{2}}\right) \frac{\sigma_{z}^{\prime}}{\sigma_{y}}
\end{aligned}
$$

The equivalent residual stress at a general radius location based on the Mises criterion is

$$
\frac{\sigma_{e}^{\prime}}{\sigma_{y}}=\frac{\sqrt{3}}{2}\left(\frac{\sigma_{\theta}^{\prime}}{\sigma_{y}}-\frac{\sigma_{r}^{\prime}}{\sigma_{y}}\right)=\frac{k^{2}\left(k_{j}^{2}-1-\ln k_{j}^{2}\right)}{\left(k^{2}-1\right) x^{2}}
$$

Since $\sigma_{e}^{\prime}=\sigma_{\theta}^{\prime}-\sigma_{r}^{\prime}$ based on the maximum shear stress theory (Tresca criterion) and $\sigma_{e}^{\prime}=(\sqrt{3} / 2)\left(\sigma_{\theta}^{\prime}-\sigma_{r}^{\prime}\right)$ based on the Mises criterion, while the components of the residual stress based on Mises criterion are $2 / \sqrt{3}$ times those based on Tresca criterion, the equivalent residual stresses based on both criterions must be the same.

At the inner surface, $x=1$. For Eq. (2.2), letting $x=1$ and $\sigma_{e}^{\prime}=-\sigma_{y}$ obtains an equation for $k_{j^{*}}$, the maximum and optimum plastic depth $\left(k_{j}\right)$ for a certain $k$ to avoid compressive yield after removing $p_{a}$

$$
k^{2} \ln k_{j^{*}}^{2}-k^{2}-k_{j^{*}}^{2}+2=0 \quad k_{j^{*}} \geqslant \sqrt{e}
$$

where $\sqrt{e} \leqslant k_{j^{*}} \leqslant k_{c}$ and $k \geqslant k_{c}$. When $k \leqslant k_{c},\left|\sigma_{e}^{\prime} / \sigma_{y}\right|<1$, irrespective of $k_{j}$, Eq. (2.5) is just the equation proposed by Zhu (2008) in another method.

\section{Residual stresses and total stresses when $p=p_{a}$}

The total stresses $\sigma^{t}$ include the residual stresses plus the stresses caused by $p$, or $\sigma^{t}=\sigma^{\prime}+\sigma^{p}$.

To produce plastic depth $k_{j}$, the pressure subjected to a cylinder is the autofrettage pressure $p_{a}$

$$
\frac{p}{\sigma_{y}}=\frac{2}{\sqrt{3}} \ln k_{j}+\frac{k^{2}-k_{j}^{2}}{\sqrt{3} k^{2}}=\frac{p_{a}}{\sigma_{y}}
$$


Letting $k=\infty$ in Eq. (3.1), one obtains

$$
\frac{p_{\infty}}{\sigma_{y}}=\frac{2}{\sqrt{3}} \ln k_{j}+\frac{1}{\sqrt{3}}
$$

Inappropriate $k_{j}$ causes compressive yield or reduces load-bearing capacity. To avoid compressive yield, the plastic depth $k_{j}$ for a certain $k$ must be smaller than or equal to the magnitude calculated by Eq. (2.5). Then, to raise load-bearing capacity fully, combining Eqs. (2.5) and (3.1), one obtains

$$
\frac{p}{\sigma_{y}}=\frac{2\left(k^{2}-1\right)}{\sqrt{3} k^{2}}=\frac{2 p_{e}}{\sigma_{y}}
$$

Equation (3.3) is the optimum load-bearing capacity of an autofrettaged cylinder, it is just two times the initial yield load. The limit of Eq. (3.3) with $k \rightarrow \infty$ is $p / \sigma_{y}=2 / \sqrt{3}$, which can be obtained by letting $k_{j}=\sqrt{e}$ in Eq. (3.2) as well.

The stresses caused by $p$ at a general radius location are

$$
\frac{\sigma_{z}^{p}}{\sigma_{y}}=\frac{1}{k^{2}-1} \frac{p}{\sigma_{y}} \quad \frac{\sigma_{r}^{p}}{\sigma_{y}}=\left(1-\frac{k^{2}}{x^{2}}\right) \frac{\sigma_{z}^{p}}{\sigma_{y}} \quad \frac{\sigma_{\theta}^{p}}{\sigma_{y}}=\left(1+\frac{k^{2}}{x^{2}}\right) \frac{\sigma_{z}^{p}}{\sigma_{y}}
$$

The equivalent stress of Eq. (3.4) based on the Mises criterion is

$$
\frac{\sigma_{e}^{p}}{\sigma_{y}}=\frac{\sqrt{3}}{2}\left(\frac{\sigma_{\theta}^{p}}{\sigma_{y}}-\frac{\sigma_{r}^{p}}{\sigma_{y}}\right)=\frac{\sqrt{3} k^{2}}{k^{2}-1} \frac{p}{\sigma_{y}} \frac{1}{x^{2}}
$$

When $p=p_{a}$, Eq. (3.5) becomes

$$
\frac{\sigma_{e}^{p}}{\sigma_{y}}=\frac{2 k^{2}}{k^{2}-1} \frac{p}{\sigma_{y}} \frac{1}{x^{2}}=\frac{k^{2}-k_{j}^{2}+k^{2} \ln k_{j}^{2}}{\left(k^{2}-1\right) x^{2}}
$$

The equivalent total stress $\sigma_{e}^{t}$ is

$$
\sigma_{e}^{t}=\frac{\sqrt{3}}{2}\left(\sigma_{\theta}^{t}-\sigma_{r}^{t}\right)=\frac{\sqrt{3}}{2}\left[\left(\sigma_{\theta}^{\prime}+\sigma_{\theta}^{p}\right)-\left(\sigma_{r}^{\prime}+\sigma_{r}^{p}\right)\right]=\frac{\sqrt{3}}{2}\left[\left(\sigma_{\theta}^{\prime}-\sigma_{r}^{\prime}\right)+\left(\sigma_{\theta}^{p}-\sigma_{r}^{p}\right)\right]=\sigma_{e}^{\prime}+\sigma_{e}^{p}
$$

Then, generally, in the plastic zone

$$
\frac{\sigma_{e}^{t}}{\sigma_{y}}=1-\frac{k^{2}-k_{j}^{2}+k^{2} \ln k_{j}^{2}}{\left(k^{2}-1\right) x^{2}}+\frac{\sqrt{3} k^{2}}{k^{2}-1} \frac{p}{\sigma_{y}} \frac{1}{x^{2}}
$$

in the elastic zone

$$
\frac{\sigma_{e}^{t}}{\sigma_{y}}=\frac{k^{2}\left(k_{j}^{2}-1-\ln k_{j}^{2}\right)}{\left(k^{2}-1\right) x^{2}}+\frac{\sqrt{3} k^{2}}{k^{2}-1} \frac{p}{\sigma_{y}} \frac{1}{x^{2}}
$$

At the elastic-plastic juncture $\left(x=k_{j}\right)$, Eqs (3.8) and (3.9) both become

$$
\frac{\sigma_{e}^{t}}{\sigma_{y}}=\frac{k^{2}\left(k_{j}^{2}-1-\ln k_{j}^{2}\right)}{\left(k^{2}-1\right) k_{j}^{2}}+\frac{\sqrt{3} k^{2} p}{\sigma_{y}\left(k^{2}-1\right) k_{j}^{2}}
$$

When $p=p_{a}$, the first one of Eq. (3.4) becomes

$$
\frac{\sigma_{z}^{p}}{\sigma_{y}}=\frac{k^{2} \ln k_{j}^{2}+k^{2}-k_{j}^{2}}{\sqrt{3} k^{2}\left(k^{2}-1\right)}
$$


Using Eqs. (3.11) and (3.4), the general residual stress, Eqs. (2.1)-(2.4), can be rewritten as follows:

— in the plastic zone

$$
\begin{aligned}
& \frac{\sigma_{z}^{\prime}}{\sigma_{y}}=\frac{1}{\sqrt{3}}\left(\frac{k_{j}^{2}}{k^{2}}+\ln x^{2}-\ln k_{j}^{2}\right)-\frac{\sigma_{z}^{p}}{\sigma_{y}} \quad \frac{\sigma_{r}^{\prime}}{\sigma_{y}}=\frac{1}{\sqrt{3}}\left(\frac{k_{j}^{2}}{k^{2}}-1+\ln x^{2}-\ln k_{j}^{2}\right)-\frac{\sigma_{r}^{p}}{\sigma_{y}} \\
& \frac{\sigma_{\theta}^{\prime}}{\sigma_{y}}=\frac{1}{\sqrt{3}}\left(\frac{k_{j}^{2}}{k^{2}}+1+\ln x^{2}-\ln k_{j}^{2}\right)-\frac{\sigma_{\theta}^{p}}{\sigma_{y}} \\
& \frac{\sigma_{e}^{\prime}}{\sigma_{y}}=\frac{\sqrt{3}}{2}\left(\frac{\sigma_{\theta}^{\prime}}{\sigma_{y}}-\frac{\sigma_{r}^{\prime}}{\sigma_{y}}\right)=1-\left(\frac{\sigma_{\theta}^{p}}{\sigma_{y}}-\frac{\sigma_{r}^{p}}{\sigma_{y}}\right)=1-\frac{\sigma_{e}^{p}}{\sigma_{y}}
\end{aligned}
$$

— in the elastic zone

$$
\begin{array}{ll}
\frac{\sigma_{z}^{\prime}}{\sigma_{y}}=\frac{1}{\sqrt{3}} \frac{k_{j}^{2}}{k^{2}}-\frac{\sigma_{z}^{p}}{\sigma_{y}} & \frac{\sigma_{r}^{\prime}}{\sigma_{y}}=\frac{1}{\sqrt{3}}\left(\frac{k_{j}^{2}}{k^{2}}-\frac{k_{j}^{2}}{x^{2}}\right)-\frac{\sigma_{r}^{p}}{\sigma_{y}} \\
\frac{\sigma_{\theta}^{\prime}}{\sigma_{y}}=\frac{1}{\sqrt{3}}\left(\frac{k_{j}^{2}}{k^{2}}+\frac{k_{j}^{2}}{x^{2}}\right)-\frac{\sigma_{\theta}^{p}}{\sigma_{y}} & \frac{\sigma_{e}^{\prime}}{\sigma_{y}}=\frac{\sqrt{3}}{2} \frac{\sigma_{\theta}^{\prime}}{\sigma_{y}}-\frac{\sigma_{r}^{\prime}}{\sigma_{y}}=\frac{k_{j}^{2}}{x^{2}}-\frac{\sqrt{3}}{2}\left(\frac{\sigma_{\theta}^{p}}{\sigma_{y}}-\frac{\sigma_{r}^{p}}{\sigma_{y}}\right)=\frac{k_{j}^{2}}{x^{2}}-\frac{\sigma_{e}^{p}}{\sigma_{y}}
\end{array}
$$

Therefore, when $p=p_{a}$, irrespective of $k_{j}$, the total stresses are:

- in the plastic zone

$$
\begin{array}{ll}
\frac{\sigma_{z}^{t}}{\sigma_{y}}=\frac{\sigma_{z}^{\prime}}{\sigma_{y}}+\frac{\sigma_{z}^{p}}{\sigma_{y}}=\frac{1}{\sqrt{3}}\left(\frac{k_{j}^{2}}{k^{2}}+\ln x^{2}-\ln k_{j}^{2}\right) & \\
\frac{\sigma_{r}^{t}}{\sigma_{y}}=\frac{\sigma_{r}^{\prime}}{\sigma_{y}}+\frac{\sigma_{r}^{p}}{\sigma_{y}}=\frac{1}{\sqrt{3}}\left(\frac{k_{j}^{2}}{k^{2}}-1+\ln x^{2}-\ln k_{j}^{2}\right) & \\
\frac{\sigma_{\theta}^{t}}{\sigma_{y}}=\frac{\sigma_{\theta}^{\prime}}{\sigma_{y}}+\frac{\sigma_{\theta}^{p}}{\sigma_{y}}=\frac{1}{\sqrt{3}}\left(\frac{k_{j}^{2}}{k^{2}}+1+\ln x^{2}-\ln k_{j}^{2}\right) & \frac{\sigma_{e}^{t}}{\sigma_{y}} \equiv 1
\end{array}
$$

— in the elastic zone

$$
\begin{array}{ll}
\frac{\sigma_{z}^{t}}{\sigma_{y}}=\frac{\sigma_{z}^{\prime}}{\sigma_{y}}+\frac{\sigma_{z}^{p}}{\sigma_{y}}=\frac{1}{\sqrt{3}} \frac{k_{j}^{2}}{k^{2}} & \frac{\sigma_{r}^{t}}{\sigma_{y}}=\frac{\sigma_{r}^{\prime}}{\sigma_{y}}+\frac{\sigma_{r}^{p}}{\sigma_{y}}=\frac{1}{\sqrt{3}}\left(\frac{k_{j}^{2}}{k^{2}}-\frac{k_{j}^{2}}{x^{2}}\right) \\
\frac{\sigma_{\theta}^{t}}{\sigma_{y}}=\frac{\sigma_{\theta}^{\prime}}{\sigma_{y}}+\frac{\sigma_{\theta}^{p}}{\sigma_{y}}=\frac{1}{\sqrt{3}}\left(\frac{k_{j}^{2}}{k^{2}}+\frac{k_{j}^{2}}{x^{2}}\right) & \frac{\sigma_{e}^{t}}{\sigma_{y}}=\frac{k_{j}^{2}}{x^{2}}
\end{array}
$$

The components of total stresses based on the Mises criterion are $2 / \sqrt{3}$ times those based on the Tresca criterion, but the equivalent total stresses based on both theories in the plastic and elastic zone are the same, respectively. The reason is that the equivalent total stress based on the Tresca criterion is $2 / \sqrt{3}$ times that based on the Mises criterion since $\sigma_{z}=\left(\sigma_{r}+\sigma_{\theta}\right) / 2$ for cylinders.

\section{Residual stresses and total stresses when $k_{j}=k_{j^{*}}$ and $p=p_{a}$}

If $k_{j}$ is determined by Eq. (2.5), or $k_{j}=k_{j^{*}}$, Eqs. (3.8) and (3.9) become respectively

$$
\frac{\sigma_{e}^{t}}{\sigma_{y}}=1-\frac{2}{x^{2}}+\frac{\sqrt{3} k^{2}}{k^{2}-1} \frac{p / \sigma_{y}}{x^{2}} \quad \frac{\sigma_{e}^{t}}{\sigma_{y}}=\frac{k_{j}^{2}-2}{x^{2}}+\frac{\sqrt{3} k^{2}}{k^{2}-1} \frac{p / \sigma_{y}}{x^{2}}
$$

From Eq. $(4.1)_{1}$, it is seen that:

(1) provided $p / \sigma_{y}>-2\left(k^{2}-1\right)\left(x^{2}-1\right) / \sqrt{3} k^{2}$ (negative), $\sigma_{e}^{t}>-\sigma_{y}$, this is definitely feasible for $p>0$ in engineering; 
(2) as long as $p / \sigma_{y}>\left(k^{2}-1\right)\left(2-x^{2}\right) / \sqrt{3} k^{2}, \sigma_{e}^{t}>0$, while $\left(k^{2}-1\right)\left(2-x^{2}\right) / \sqrt{3} k^{2}<p_{e} / \sigma_{y}$, so when $p>p_{e}, \sigma_{e}^{t}>0$;

(3) so long as $p<2 p_{e}, \sigma_{e}^{t}<\sigma_{y}$. Thus, when $p_{e}<p<2 p_{e}, 0<\sigma_{e}^{t}<\sigma_{y}$.

From Eq. $(4.1)_{2}$, it is known that:

(1) provided $p / \sigma_{y}>-\left(k^{2}-1\right)\left(k_{j}^{2}-2\right) / \sqrt{3} k^{2}$ (negative), $\sigma_{e}^{t}>0$, this is certain for $p>0$ in engineering, so the equivalent residual stress in the elastic zone is always tensile;

(2) so long as $p / \sigma_{y}<\left(k^{2}-1\right)\left(x^{2}-k_{j}^{2}+2\right) / \sqrt{3} k^{2}, \sigma_{e}^{t}<\sigma_{y}$, so when $p<2 p_{e}, \sigma_{e}^{t}<\sigma_{y}$.

At the inside surface, $x=1$, then, from Eq. $(4.1)_{1}$

$$
\frac{\sigma_{e}^{t}}{\sigma_{y}}=\frac{\sqrt{3} k^{2}}{k^{2}-1} \frac{p}{\sigma_{y}}-1
$$

Unless $p<0, \sigma_{e}^{t}$ can not be lower than $-\sigma_{y}$. Unless $p>2 p_{e}, \sigma_{e}^{t}$ can not be higher than $\sigma_{y}$. So, when $0<p<2 p_{e},-1<\sigma_{e}^{t} / \sigma_{y}<1$. Especially, when $p=2 p_{e}, \sigma_{e}^{t} \equiv \sigma_{y}$ in the whole plastic zone.

At the elastic-plastic juncture, $x=k_{j}$, from $(4.1)_{1}$ or $(4.1)_{2}$

$$
\frac{\sigma_{e}^{t}}{\sigma_{y}}=\frac{k_{j}^{2}-2}{k_{j}^{2}}+\frac{\sqrt{3} k^{2}}{k^{2}-1} \frac{p / \sigma_{y}}{k_{j}^{2}}
$$

Clearly, $\sigma_{e}^{t}>0$ in the elastic zone. If $p<2 p_{e}, \sigma_{e}^{t}$ can not be higher than $\sigma_{y}$. So, when $0<p<2 p_{e}, 0<\sigma_{e}^{t} / \sigma_{y}<1$. Especially, when $p=2 p_{e}, \sigma_{e}^{t}=\sigma_{y}$ at the elastic-plastic juncture and $\sigma_{e}^{t} / \sigma_{y}=k_{j}^{2} / x^{2}$ at a general radius location in the elastic zone.

When $k_{j}=k_{j^{*}}$, by the aid of Eq. (2.5), Eqs (2.1)-(2.4) can be simplified as follows:

- in the plastic zone

$$
\begin{array}{ll}
\frac{\sigma_{z}^{\prime}}{\sigma_{y}}=\frac{\ln x^{2}}{\sqrt{3}}-\frac{1}{\sqrt{3}} & \frac{\sigma_{r}^{\prime}}{\sigma_{y}}=\frac{\ln x^{2}}{\sqrt{3}}+\frac{2}{\sqrt{3} x^{2}}-\frac{2}{\sqrt{3}} \\
\frac{\sigma_{\theta}^{\prime}}{\sigma_{y}}=\frac{\ln x^{2}}{\sqrt{3}}-\frac{2}{\sqrt{3} x^{2}} & \frac{\sigma_{e}^{\prime}}{\sigma_{y}}=1-\frac{2}{x^{2}}
\end{array}
$$

— in the elastic zone

$$
\begin{array}{ll}
\frac{\sigma_{z}^{\prime}}{\sigma_{y}}=\frac{k_{j}^{2}-2}{\sqrt{3} k^{2}} & \frac{\sigma_{r}^{\prime}}{\sigma_{y}}=\left(1-\frac{k^{2}}{\left(r / r_{i}\right)^{2}}\right) \frac{\sigma_{z}^{\prime}}{\sigma_{y}}=\left(1-\frac{k^{2}}{x^{2}}\right) \frac{\sigma_{z}^{\prime}}{\sigma_{y}} \\
\frac{\sigma_{\theta}^{\prime}}{\sigma_{y}}=\left(1+\frac{k^{2}}{x^{2}}\right) \frac{\sigma_{z}^{\prime}}{\sigma_{y}} & \frac{\sigma_{e}^{\prime}}{\sigma_{y}}=\frac{k_{j}^{2}-2}{x^{2}}
\end{array}
$$

The equations of residual stresses are greatly simplified, and cylinders are safe after removing $p_{a}$.

When $k=\infty, k_{j}=\sqrt{e}$, from Eq. (4.5) $)_{4}$, the equivalent residual stress at a general radius location in the elastic zone is

$$
\frac{\sigma_{e}^{\prime}}{\sigma_{y}}=\frac{e-2}{x^{2}} \quad \frac{\sigma_{e}^{\prime}}{\sigma_{y}}=1-\frac{2}{e} \rightarrow 0 \quad \text { when } \quad x=\sqrt{e} \rightarrow \infty
$$

When $k_{j}=k_{j^{*}}$, the distributions of equivalent residual stresses in the plastic and elastic zones - which are the same as those based on Tresca criterion - are illustrated in Fig. 1. In Fig. 1:

(1) Curve $B A A: k=k_{j}=k_{c}, x$ varies from 1 to $k_{j}$ in the plastic zone (from point $B$ to $A$ ), and from $2.2184574899167 \ldots\left(k_{j}\right)$ to $2.2184574899167 \ldots(k)$ (from point $A$ to $A$ ) in the elastic zone (no elastic zone). 
(2) Curve $B C D: k=2.25, k_{j}=2.046308 \ldots, x$ varies from 1 to $2.046308 \ldots$ in the plastic zone (from point $B$ to $C$ ), and from $2.046308 \ldots$ to 2.25 (from point $C$ to $D$ ) in the elastic zone.

(3) Curve $B E F: k=3, k_{j}=1.748442 \ldots, x$ varies from 1 to $1.748442 \ldots$ in the plastic zone (from point $B$ to $E$ ), and from $1.748442 \ldots$ to 3 (from point $E$ to $F$ ) in elastic zone.

(4) Curve $B M N: k=\infty, k_{j}=\sqrt{e}, x$ varies from 1 to $\sqrt{e}=1.648721 \ldots$ in the plastic zone (from point $B$ to $M$ ), and from $\sqrt{e}$ to $k=\infty$ (from point $M$ to $N$ ) in the elastic zone.

The above results and Fig. 1 are fit for both the Tresca and Mises criterion.

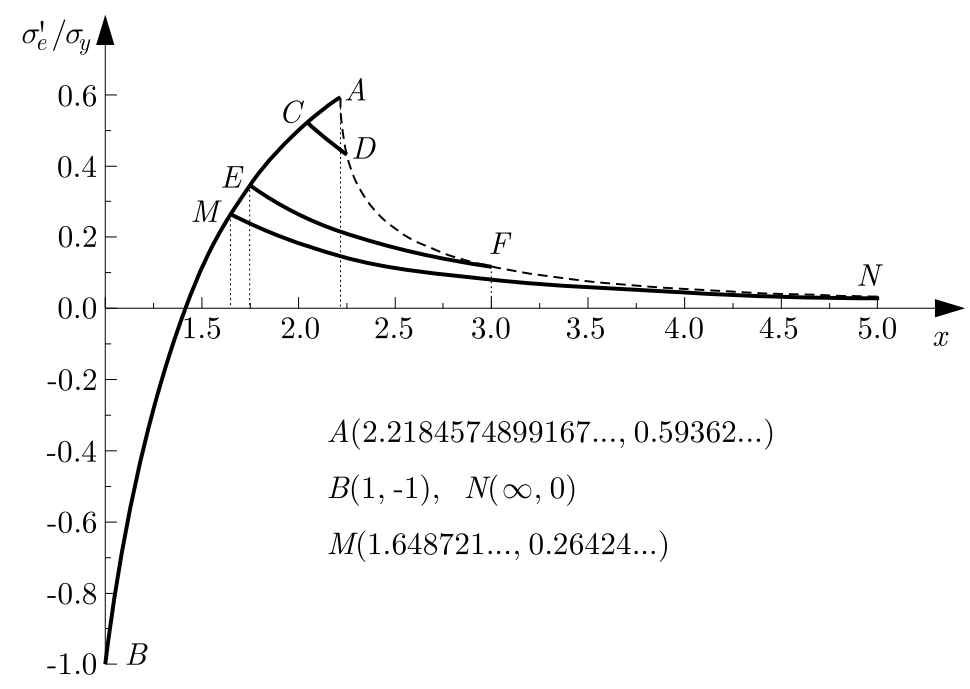

Fig. 1. The distributions of equivalent residual stresses in the whole wall

Figure 1 and Eq. (4.4) 4 show that all curves of equivalent residual stresses for any $k$ and $k_{j}$ in the plastic zone are located on the identical curve $A B$ and pass through the same point $(\sqrt{2}, 0)$, except that a different curve for different $k$ and $k_{j}$ is located in a different section of the curve $A B$, i.e. curves $B A, B C, B E$ and $B M$ are all on the curve $B A$ or they coincide with each other. However, if $k_{j} \neq k_{j^{*}}$ or the relation between $k_{j}$ and $k$ does not satisfy Eq. (2.5), the above argument is untenable, or even $\left|\sigma_{e}^{\prime}\right|>\sigma_{y}$. This case is illustrated in Fig. 2, where the curves $B E F$ and $B K L$ coincide with each other in the plastic zone and both pass through point $(\sqrt{2}, 0)$ because $k_{j}=k_{j^{*}}$, but curves $H S I$ and GQJ neither coincide with each other in the plastic zone nor pass through the point $(\sqrt{2}, 0)$, and they do not coincide with the curves $B E F$ and $B K L$ in the plastic zone for $k_{j} \neq k_{j^{*}}$.

When $p=2 p_{e}$, Eq. (3.4) and (3.5) become Eq. (4.7) and Eq. (4.8), respectively

$$
\frac{\sigma_{z}^{p}}{\sigma_{y}}=\frac{2}{\sqrt{3} k^{2}} \quad \frac{\sigma_{r}^{p}}{\sigma_{y}}=\frac{2}{\sqrt{3} k^{2}}-\frac{2}{\sqrt{3} x^{2}} \quad \frac{\sigma_{\theta}^{p}}{\sigma_{y}}=\frac{2}{\sqrt{3} k^{2}}+\frac{2}{\sqrt{3} x^{2}}
$$

and

$$
\frac{\sigma_{e}^{p}}{\sigma_{y}}=\frac{2}{x^{2}}
$$

When $k_{j}=k_{j^{*}}, p=p_{a}=2 p_{e}$, thus, when $p=p_{a}=2 p_{e}$, Eqs. (3.14) and (3.15) for the total stresses become:

— in the plastic zone

$$
\begin{array}{ll}
\frac{\sigma_{z}^{t}}{\sigma_{y}}=\frac{\ln x^{2}}{\sqrt{3}}-\frac{1}{\sqrt{3}}+\frac{2}{\sqrt{3} k^{2}} & \frac{\sigma_{r}^{t}}{\sigma_{y}}=\frac{\ln x^{2}}{\sqrt{3}}-\frac{2}{\sqrt{3}}+\frac{2}{\sqrt{3} k^{2}} \\
\frac{\sigma_{\theta}^{t}}{\sigma_{y}}=\frac{\ln x^{2}}{\sqrt{3}}+\frac{2}{\sqrt{3} k^{2}} & \frac{\sigma_{e}^{t}}{\sigma_{y}}=\frac{\sqrt{3}}{2}\left(\frac{\sigma_{\theta}^{t}}{\sigma_{y}}-\frac{\sigma_{r}^{t}}{\sigma_{y}}\right) \equiv 1
\end{array}
$$




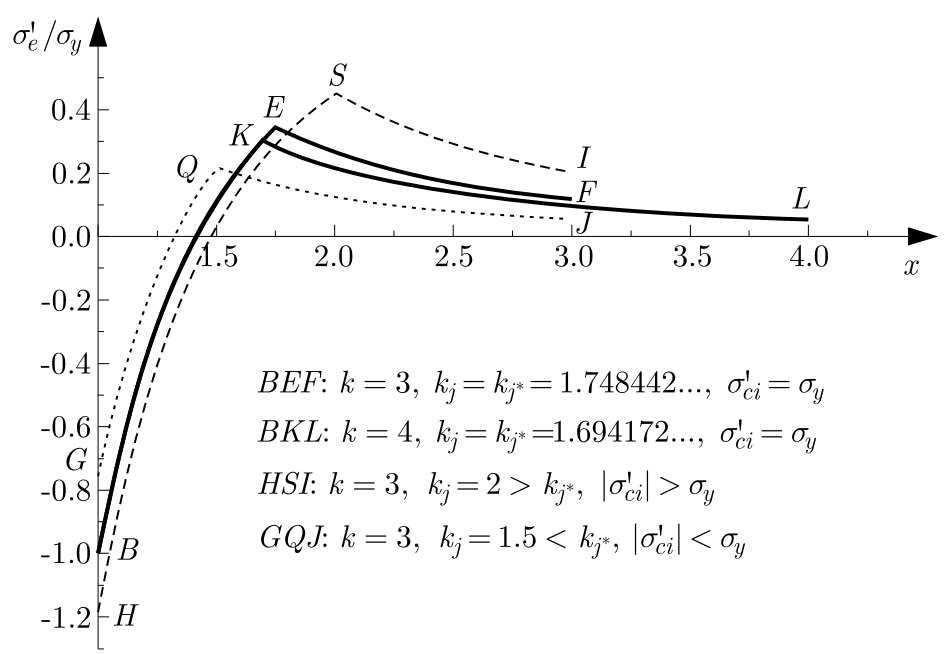

Fig. 2. Comparison between equivalent residual stresses for different $k$ and $k_{j}$

— in the elastic zone

$$
\begin{aligned}
& \frac{\sigma_{z}^{t}}{\sigma_{y}}=\frac{\sigma_{z}^{\prime}}{\sigma_{y}}+\frac{\sigma_{z}^{p}}{\sigma_{y}}=\frac{k_{j}^{2}}{\sqrt{3} k^{2}} \quad \frac{\sigma_{r}^{t}}{\sigma_{y}}=\frac{\sigma_{r}^{\prime}}{\sigma_{y}}+\frac{\sigma_{r}^{p}}{\sigma_{y}}=\frac{k_{j}^{2}}{\sqrt{3}}\left(\frac{1}{k^{2}}-\frac{1}{x^{2}}\right) \\
& \frac{\sigma_{\theta}^{t}}{\sigma_{y}}=\frac{\sigma_{\theta}^{\prime}}{\sigma_{y}}+\frac{\sigma_{\theta}^{p}}{\sigma_{y}}=\frac{k_{j}^{2}}{\sqrt{3}}\left(\frac{1}{k^{2}}+\frac{1}{x^{2}}\right) \quad \frac{\sigma_{e}^{t}}{\sigma_{y}}=\frac{k_{j}^{2}}{x^{2}} \quad \frac{\sigma_{e}^{t}}{\sigma_{y}}=\frac{e}{x^{2}}
\end{aligned}
$$

where $k=\infty, x \in(\sqrt{e}, \infty)$.

The equations of total stresses are greatly simplified, and cylinders are safe after removing $p_{a}$ and in operation. Figure 3 shows the distribution of equivalent stress of the total stress when $p=2 p_{e}$ and $k_{j}=k_{j^{*}}$. In Fig. 3:

(1) Horizontal line baa: $k=k_{j}=k_{c}$. In the plastic zone, $\sigma_{e}^{t} / \sigma_{y}$ is a horizontal line: $\sigma_{e}^{t} / \sigma_{y}=1$, $x$ varies from 1 to $k_{j}$ (from point $b$ to $a$ ) and from $k_{j}$ to $k$ (from point $a$ to $a$ ) in the plastic zone (no elastic zone).

(2) Curve $b c d: k=2.25, k_{j}=2.046308 \ldots$. In the plastic zone, $\sigma_{e}^{t} / \sigma_{y}$ is a horizontal line, $b c: \sigma_{e}^{t} / \sigma_{y}=1, x$ varies from 1 to $k_{j}$ (from point $b$ to $c$ ) and from $k_{j}$ to $k$ (from point $c$ to $d$ ) in the elastic zone.

(3) Curve bef: $k=3, k_{j}=1.748442 \ldots$ In the plastic zone, $\sigma_{e}^{t} / \sigma_{y}$ is a horizontal line, be: $\sigma_{e}^{t} / \sigma_{y}=1, x$ varies from 1 to $k_{j}$ (from point $b$ to $e$ ) and from $k_{j}$ to $k$ (from point $e$ to $f$ ) in the elastic zone.

(4) Curve bmn: $k=\infty, k_{j}=\sqrt{e}$. In the plastic zone, $\sigma_{e}^{t} / \sigma_{y}$ is a horizontal line: $\sigma_{e}^{t} / \sigma_{y}=1$, $b m: x$ varies from 1 to $k_{j}$ (from point $b$ to $m$ ) and from $k_{j}$ to $k(\infty)$ (from point $m$ to $n$ ) in the elastic zone.

If $k_{j} \neq k_{j^{*}}$ or $p \neq 2 p_{e}$, the above traits can not arise. Figure 4 is comparison between the equivalent total stresses under different internal pressure and $k_{j}=k_{j^{*}}$ from which it is known that only when $p=2 p_{e}$ and $k_{j}=k_{j^{*}}$, the operation state is optimum, otherwise, or $p \neq 2 p_{e}$ and/or $k_{j} \neq k_{j^{*}}$, either $\sigma_{e}^{t}>\sigma_{y}$ or load-bearing capacity is lowered or compressive yield occurs. In Fig. 4, curve 1 is just curve bef in Fig. 3.

Besides, for a certain $k$, when $k_{j}<k_{j^{*}}$, though residual stresses are lower than those when $k_{j}=k_{j^{*}}$, the load-bearing capacity is dropped. For example, for $k=3$, if $k_{j}=k_{j^{*}}(=1.748442)$, from Eq. (3.3), $p / \sigma_{y}=1.0264 \ldots$; while if $k_{j}=1.5$, from Eq. (3.1), $p / \sigma_{y}=0.901203 \ldots<1.0264$. 


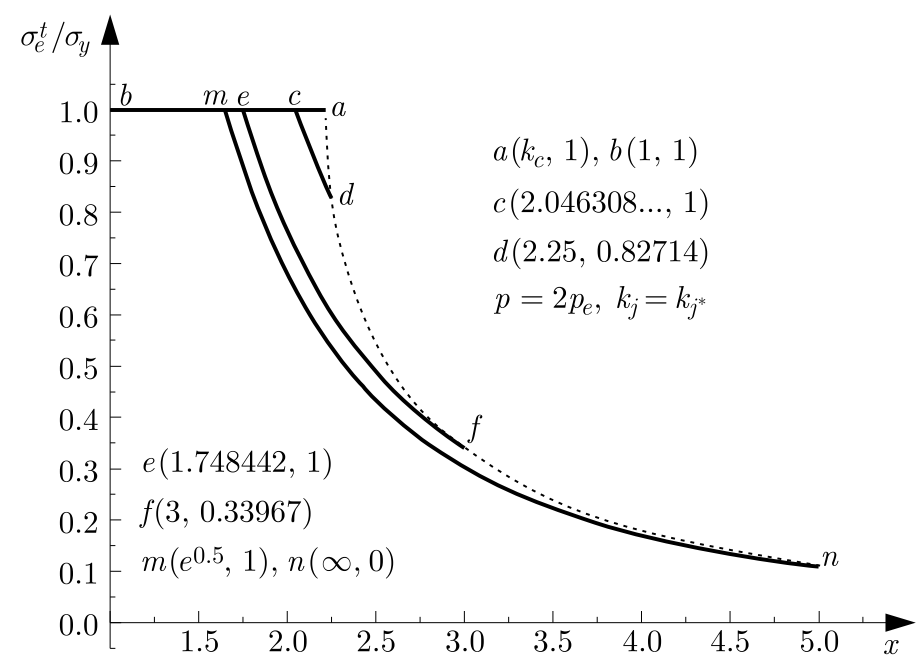

Fig. 3. The distribution of equivalent total stress when $p=2 p_{e}$ and $k_{j}=k_{j^{*}}$

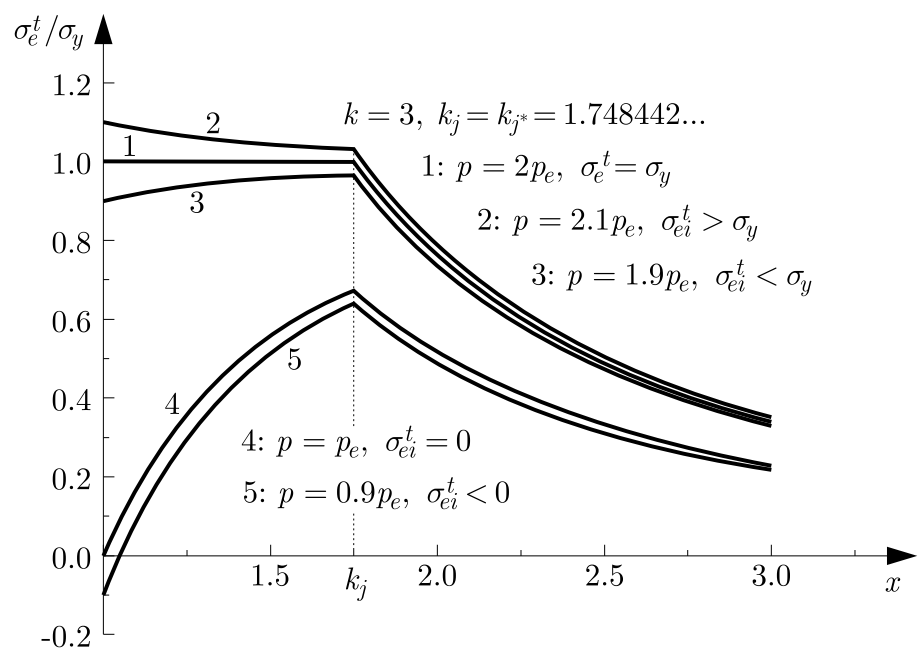

Fig. 4. Comparison between the equivalent total stresses for different internal pressure and $k_{j}=k_{j^{*}}$

\section{Conclusions}

It is the combination of $k^{2} \ln k_{j^{*}}^{2}-k^{2}-k_{j^{*}}^{2}+2=0$ and $p=2 p_{e}$ that results in optimum results of an autofrettaged cylinder - the load-bearing capacity is the highest and no compressive yield occurs after removing $p_{a}$. Under these two conditions, neither compressive yield occurs nor do the equivalent total stresses exceed $\sigma_{y}$, and the equivalent total stress in the whole plastic zone is even, identically equal to $\sigma_{y}$, while the equivalent total stress in the elastic zone is lower than $\sigma_{y}$.

The equations concerned with autofrettage are simplified greatly because of the conditions $k^{2} \ln k_{j^{*}}^{2}-k^{2}-k_{j^{*}}^{2}+2=0$ and $p=2 p_{e}$.

When $k_{j}=k_{j^{*}}$, in the plastic zone, the equivalent residual stress $\sigma_{e}^{\prime} / \sigma_{y}$ shares the same curve and pass through the point $(\sqrt{2}, 0)$. When $x \leqslant \sqrt{2}, \sigma_{e}^{\prime} \leqslant 0$; when $x \geqslant \sqrt{2}, \sigma_{e}^{\prime} \geqslant 0$. The equivalent residual stress at the inside surface $\sigma_{e i}^{\prime}=-\sigma_{y}$; in the whole wall, $\left|\sigma_{e}^{\prime}\right| \leqslant \sigma_{y}$. So when $k_{j}=k_{j^{*}}$, cylinders are safe.

When $p>2 p_{e}$ and $k_{j}=k_{j^{*}}$, the equivalent total stress is greater than $\sigma_{y}$ and uneven. When $p<2 p_{e}$ and $k_{j}=k_{j^{*}}$, the equivalent total stress is lower than $\sigma_{y}$ but load-bearing capacity is reduced, and equivalent total stress is uneven. When $p<p_{e}, \sigma_{e}^{t}$ at the inside surface is lower 
than $0, \sigma_{e}^{t}$ can not be lower than $-\sigma_{y}$ in the whole plastic zone, the load-bearing capacity is reduced greatly and the equivalent total stress is uneven.

As long as an autofrettaged cylinder contains the autofrettage pressure $p_{a}$, there must be an inexorable law irrespective of $k_{j}$ and $k: \sigma_{e} / \sigma_{y} \equiv 1$ in the plastic zone and $0<\sigma_{e} / \sigma_{y}=k_{j}^{2} / x^{2}<1$ in the elastic zone. Nevertheless, too great $k_{j}$ causes compressive yield after removing $p_{a}$, too small $k_{j}$ reduces the load-bearing capacity of a cylinder. The optimum plastic depth is $k_{j}=k_{j^{*}}$, and when $k_{j}=k_{j^{*}}, p=p_{a}=2 p_{e}$.

On the basis of the results in this study, when an autofrettaged cylinder is subjected to the load $p=2 p_{e}=(2 / \sqrt{3})\left[\left(k^{2}-1\right) / k^{2}\right] \sigma_{y}$, its optimum design thickness is

$$
t=r_{i}(k-1)=r_{i}\left(\sqrt{\frac{2 \sigma_{y}}{\sigma_{y}-\sqrt{3} p}}-1\right)
$$

its optimum plastic depth $k_{j^{*}}$ is calculated by

$$
\frac{2 \sigma_{y}}{\sigma_{y}-\sqrt{3} p} \ln k_{j *}^{2}-\frac{2 \sigma_{y}}{\sigma_{y}-\sqrt{3} p}-k_{j *}^{2}+2=0
$$

\section{Acknowledgments}

This project has been supported by Scientific Research Fund of Hunan Provincial Education Department (Grant No. 12A087)

\section{References}

1. Bhatnagar R.M., 2013, Modelling, validation and design of autofrettage and compound cylinder, European Journal of Mechanics-A Solids, 39, 17-25

2. Brünnet H., BäHre D., 2014, Full exploitation of lightweight design potentials by generating pronounced compressive residual stress fields with hydraulic autofrettage, Advanced Materials Research, 907, 17-27

3. Farrahi G.H., Voyiadjis G.Z., Hoseini S.H., Hosseinian E., 2012, Residual stress analyses of re-autofrettaged thick-walled tubes, International Journal of Pressure Vessels and Piping, 98, $57-64$

4. Gibson M.C., Hameed A., Hetherington J.G., 2014, Investigation of residual stress development during swage autofrettage, using finite element analysis, Journal of Pressure Vessel Technology, 136, 2, 45-51

5. Hamilton N.R., Wood J., Easton D., Robbie M.B.O., Zhang Y., Galloway A., 2015, Thermal autofrettage of dissimilar material brazed joints, Materials an Design, 67, 405-412

6. Lin Y., Dong Q., JiA J., 2009, Autofrettage damage residual stress of thick-walled cylinder (in Chinese), Science Technology and Engineering, 9, 24, 7306-7309

7. Lvov G., Kostromitskaya O., 2014, Effect of material damage on autofrettage of thick-walled cylinder, Universal Journal of Mechanical Engineering, 2, 2, 44-48

8. Noraziah W., Amran A., Elbasheer M.K., 2011a, Effect of autofrettage on allowable pressure of thick-walled cylinders, Proceedings of International Conference on Environmental and Agriculture Engineering (ICEAE 2011), Singapore

9. Noraziah W., Amran A., Elbasheer M.K., 2011b, Effect of optimum autofrettage on pressure limits of thick-walled cylinder, International Journal of Environmental Science and Development, $\mathbf{2}, 4,329-333$

10. Shim W.S., Kim J.H., Lee Y.S., Cha K.U., Hong K.S., 2010, A Study on hydraulic autofrettage of thick-walled cylinders incorporating Bauschinger effect, Experimental Mechanics, 50, 5, 621-626 
11. Yu G., 1980, Chemical Pressure Vessels and Equipment (in Chinese), Beijing: Chemical Industrial Press

12. Zheng X.T., XuAn F.Z., 2010, Investigation on autofrettage and safety of the thick-walled cylinder under thermo-mechanical loadings (in Chinese), Journal of Mechanical Engineering, 46, 16, 156-161

13. Zheng X.T., XuAn F.Z., 2011, Autofrettage and shakedown analysis of strain-hardening cylinders under thermo-mechanical loadings, Journal of Strain Analysis Engineering and Design, 46, 1, 45-55

14. ZHU R., 2008, Ultimate load-bearing capacity of cylinder derived from autofrettage under ideal condition, Chinese Journal of Mechanical Engineering, 21, 5, 80-87

15. ZHu R., Li Q., 2014, Inquire into the marvellousness of autofrettage for mono-layered cylinders, Journal of Theoretical and Applied Mechanics, 52, 2, 359-372

16. ZHu R., ZHU G., 2013, On autofrettage of cylinders by limiting circumferential residual stress based on Mises yield criterion, Journal of Theoretical and Applied Mechanics, 51, 3, 697-710

Manuscript received November 4, 2015; accepted for print April 5, 2016 